\title{
Keratin 6A gene silencing suppresses cell invasion and metastasis of nasopharyngeal carcinoma via the $\beta$-catenin cascade
}

\author{
CHUANJUN $\mathrm{CHEN}^{1}$ and HUIGUO SHAN ${ }^{2}$ \\ ${ }^{1}$ Oncology Department, Xinchang People's Hospital, Shaoxing, Zhejiang 312500; \\ ${ }^{2}$ Oncology Department, The Affiliated Dongtai Hospital of Nantong University, Dongtai, Jiangsu 224200, P.R. China
}

Received June 4, 2018; Accepted March 1, 2019

DOI: $10.3892 / \mathrm{mmr} .2019 .10055$

\begin{abstract}
Nasopharyngeal carcinoma (NPC) is a type of head and neck cancer. This study aimed to study the mechanisms of ectopic keratin 6A (KRT6A) in NPC. Reverse transcription-quantitative polymerase chain reaction (RT-qPCR) and western blotting were performed to detect KRT6A levels in NPC cell lines (C666-1, 5-8F and SUNE-1) and a nasopharyngeal epithelial cell line (NP69, as a control). After SUNE-1 NPC cells had been silenced by KRT6A, cell viability, metastasis and invasion were determined using Cell Counting Kit-8, wound healing and Transwell assays, respectively. KRT6A levels, metastasis-associated factors and the Wnt/ $\beta$-catenin pathway were measured using RT-qPCR and western blotting. It was demonstrated that KRT6A was upregulated in all detected NPC cells, among which KRT6A was the highest in SUNE-1 cells. In SUNE-1 cells, cell viability was inhibited at 24 and $48 \mathrm{~h}$, and that cell metastasis and invasion were demonstrated to be suppressed by KRT6A silencing. Both the mRNA and protein levels of KRT6A, matrix metalloproteinase (MMP)-2, MMP-9, $\beta$-catenin, lymphoid enhancer binding factor 1 and T-cell specific factor 4 were reduced in the small interfering (si)KRT6A group. However, the results demonstrated that the levels of epithelial-cadherin and tissue inhibitor of metalloproteinase-2 (TIMP-2) were promoted in the siKRT6A group. The activation of the $\mathrm{Wnt} / \beta$-catenin pathway by lithium chloride reversed the effect of si-KRT6A by modulating the expression of MMP-2/9 and TIMP2. It was observed that KRT6A silencing suppressed cell invasion and metastasis of NPC via the $\beta$-catenin cascade. Together these results provide important insights into a novel approach for the diagnosis and treatment of NPC.
\end{abstract}

Correspondence to: Dr Huiguo Shan, Oncology Department, The Affiliated Dongtai Hospital of Nantong University, 2 West Kangfu Road, Dongtai, Jiangsu 224200, P.R. China

E-mail: shanhg_ghs@163.com

Key words: keratin 6A, cell invasion, cell metastasis, nasopharyngeal carcinoma, $\beta$-catenin

\section{Introduction}

Nasopharyngeal carcinoma (NPC) is a type of head and neck cancer $(1,2)$. The incidence of NPC is the highest near the parts of or in the ear, nose and throat malignancies. The incidence of NPC has obvious regional clusters and certain ethnic groups are likely to experience a higher incidence of NPC than others. Incidence is low in most areas of the world, generally below $1 / 10^{5}$ (3). However, in China NPC is mainly distributed in southern China and Southeast Asia (4). The onset ages of NPC are mostly between 40-60 years old, with males having a higher incidence compared with their female counterparts (5). The etiology of NPC includes viral infections, genetic factors, environmental and dietary factors as well as the interaction of various oncogenes and tumor suppressor genes (6). Almost $70 \%$ of NPC patients are diagnosed at the advanced stages and the five-year survival rates are lower than $10-40 \%$ (7). The main factors contributing to the failure of treatment are the characteristics of tumor recurrence and distant metastasis of NPC (8). Therefore, it is becoming increasingly significant to study how to reduce distant metastasis so as to improve the outcome of treating NPC (9).

The epithelial-mesenchymal transition (EMT) process is prevalent during tumor metastasis. EMT is characterized by weakened intercellular adhesion and increased metastasis associated factors, for example, matrix metalloproteinases (MMPs) (10,11). Tumor cells with EMT frequently manifest invasive phenotypes, which are associated with difficulty treating the tumor $(12,13)$. Notably, a couple of studies have focused on how biomarkers regulate EMT in NPC $(14,15)$. In addition, the aberrant activation of the $\mathrm{Wnt} / \beta$-catenin pathway is commonly reported to be involved in the EMT process of NPC (16).

In recent years, research on NPC in association with tumor biomarkers has attracted much attention and such studies aid early diagnosis, treatment efficacy and prognosis of NPC (17-20). Keratin is a major component of the intermediate filament protein family and is also a major structural protein of epidermal, and hair keratinocytes (21). The expression of keratin is mainly in epithelial tissues $(22,23)$. Understanding the expression of keratin genes and their normal function are the basis for maintaining the stability and normal differentiation of epidermal cells (24). A previous study demonstrated that keratin may also function to regulate cell growth and apoptosis as well as protecting cells from impairment and 
non-mechanical stress (25). Keratin 6A (KRT6A) is a family member of keratin proteins and can lead to epidermalization of squamous epithelium $(26,27)$. KRT6A is a biomarker that is unique to squamous cells (28), while NPC is an undifferentiated squamous cell carcinoma $(29,30)$. However, the correlation of KRT6A and NPC still remains unclear.

In the present study, the KRT6A silencing cell model was constructed in order to investigate the association between KRT6A and EMT in NPC. Taken from the perspective of tumor metastasis, this study help further understand the formation of NPC, aiming to improve the diagnostic compliance rate and survival rate of NPC.

\section{Materials and methods}

Cell culture. Human nasopharyngeal epithelial cell line (NP69, as a control) and NPC cell lines (C666-1, 5-8F and SUNE-1) were purchased from the American Type Culture Collection (Manassas, MA, USA), and cultured in $5 \% \mathrm{CO}_{2}$ at $37^{\circ} \mathrm{C}$. Dulbecco's modified Eagle's medium (DMEM) was used as basic culture medium. 10\% fetal bovine serum (FBS), $100 \mathrm{U} / \mathrm{ml}$ streptomycin and $100 \mu \mathrm{g} / \mathrm{ml}$ penicillin were then added in to the medium. DMEM, FBS, streptomycin and penicillin were purchased from Biological Industries (Kibbutz Beit Haemek, Israel). Lithium chloride ( $\mathrm{LiCl} ; 20 \mathrm{mM}$; incubation time $24 \mathrm{~h}$ ) was purchased from Sigma-Aldrich (Merck KGaA, Darmstadt, Germany) and was used to activate the Wnt signaling pathway as previously described (31).

Small interfering (si)RNA transfection. The well-differentiated SUNE-1 cells were evenly plated in 6-well plates at the initial concentration of $6 \times 10^{4}$ cells/well. After being cultured for $12 \mathrm{~h}$, the cells reached $60-70 \%$ confluence. The cells were cultured in DMEM without FBS. A mixture of 50 pmol KRT6A-siRNA (Shanghai GeneChem Co., Ltd., Shanghai, China), with the sequence of sense 5'-CCAGCA GGAAGAGCUAUA-3', and antisense 3'-GGUCGUCCU UCUCGAUAUU, and transfection reagent Lipofectamine ${ }^{\circledR}$ 3000 (Invitrogen; Thermo Fisher Scientific, Inc., Waltham, MA, USA) (2:1), or 50 pmol scrambled siRNA (as control; Shanghai GeneChem Co., Ltd.), with the sequence of sense: 5'-UUCUCCGAACGUGUCACGUTT-3', and antisense: 5'-ACGUGACACGUUCGGAGAA-3' in mixture with Lipofectamine ${ }^{\circledR} 3000$ was respectively added to the cells, and cultured for $8 \mathrm{~h}$ at $37^{\circ} \mathrm{C}$. The transfected cells were accordingly named the siKRT6A group and the mock group. Cells with non-treatment were named the Cotl group and treated as the control. The cells were then cultured in DMEM with FBS for another $48 \mathrm{~h}$ before further measurement of activity.

Cell viability assay. The effect of KRT6A silencing on cell viability of NPC SUNE-1 cells was measured using Cell Counting Kit-8 (CCK-8; Beyotime Institute of Biotechnology, Haimen, China) assay. To be more specific, SUNE-1 cells transfected with KRT6A-siRNA or scrambled-siRNA, or untreated SUNE-1 cells were respectively seeded in 96-well plates at an initial density of $2 \times 10^{3}$ cells/well and incubated at $37^{\circ} \mathrm{C}$ for 12,24 , and $48 \mathrm{~h}$. Next, $20 \mu \mathrm{l} \mathrm{CCK}-8$ reagent was added to each well. Then the plate was incubated at $37^{\circ} \mathrm{C}$ for $3 \mathrm{~h}$. Finally, optical density values were read at $450 \mathrm{~nm}$ using a microplate reader (BioTek Instruments, Inc., Winooski, VT, USA). The ratios of cell viabilities to Cotl group at $0 \mathrm{~h}$ were compared.

Cell scratch wound healing assay. The effect of KRT6A silencing on cell metastasis ability of NPC SUNE-1 cells was measured using wound healing assay. To be more specific, SUNE-1 cells transfected with KRT6A-siRNA or scrambled-siRNA, or SUNE-1 cells without transfection were respectively seeded in 6-well plates at an initial density of $6 \times 10^{4}$ cells/well. After reaching $80 \%$ confluence, a wound was created using pipette tip scratching. After being cultured for $24 \mathrm{~h}$, the wound closure size images in various fields were captured and measured by light microscope.

Transwell assay. Cell invasion abilities were measured using Transwell plates with $8-\mu \mathrm{m}$ pore filter (Corning, Inc., Corning, NY, USA) coated with Matrigel (BD Biosciences; Becton, Dickinson and Company, Franklin Lakes, NJ, USA). Cells $\left(6 \times 10^{4}\right.$ cells/well) suspended in serum-free medium were seeded in the upper-chambers, while medium with $20 \%$ FBS was added to the lower-chambers in order to induce cell invasion. After being cultured at $37^{\circ} \mathrm{C}$ for $24 \mathrm{~h}$, the cells were stained with crystal violet (Beyotime Institute of Biotechnology) for $15 \mathrm{~min}$ at room temperature. Finally, cells images were captured and invaded cell numbers were counted under a light microscope.

Reverse transcription-quantitative polymerase chain reaction $(R T-q P C R)$. The mRNA expression levels of KRT6A in NP69 nasopharyngeal epithelial cells and different NPC cell lines (C666-1, 5-8F and SUNE-1) were determined. After conducting KRT6A silencing, levels of KRT6A, E-cadherin, tissue inhibitor of metalloproteinase-2 (TIMP-2), MMP-2, MMP-9, $\beta$-catenin, lymphoid enhancer binding factor 1 (LEF-1) and T-cell specific factor 4 (TCF-4) were determined in the Cotl, mock and siKRT6A cell groups. Primers used are listed in Table I and GAPDH was treated as an internal control. The process was performed as follows: Total RNA was first extracted using the TRIzol reagent (Invitrogen; Thermo Fisher Scientific, Inc.) and then reverse transcribed to cDNA using Transcriptor Universal cDNA Master (Roche Diagnostics, Indianapolis, IN, USA), with the protocol of $70^{\circ} \mathrm{C}$ for $5 \mathrm{~min}$ and $42^{\circ} \mathrm{C}$ for $60 \mathrm{~min}$. Then, the PCR amplification process was conducted using LightCycler ${ }^{\circledR}$ Multiplex Masters (Roche Diagnostics) with LightCycler ${ }^{\circledR}$ 480II System (Roche Diagnostics) under the conditions as follows: Pre-denaturation at $95^{\circ} \mathrm{C}$ for $2 \mathrm{~min}, 36$ cycles (denaturation at $95^{\circ} \mathrm{C}$ for $25 \mathrm{sec}$, annealing at $60^{\circ} \mathrm{C}$ for $25 \mathrm{sec}$, extension at $72^{\circ} \mathrm{C}$ for $30 \mathrm{sec}$ ) and a final extension at $72^{\circ} \mathrm{C}$ for $10 \mathrm{~min}$. The changes were calculated by the $2^{-\Delta \Delta \mathrm{Cq}} \operatorname{method}(32)$.

Western blotting. The protein levels of KRT6A were detected in NP69 nasopharyngeal epithelial cells and in the different NPC cell lines (C666-1, 5-8F and SUNE-1). After conducting KRT6A silencing, levels of KRT6A, E-cadherin, TIMP-2, MMP-2, MMP-9, $\beta$-catenin, LEF-1 and TCF-4 were measured in Cotl, mock and siKRT6A cell groups. The cells were lysed on ice using radio immunoprecipitation assay lysis buffer (Wuhan Boster Biological Technology, Ltd., Wuhan, China) 
Table I. Primer sequences used in the reverse transcriptionquantitative polymerase chain reaction.

\begin{tabular}{|c|c|c|}
\hline Name & Type & Sequence $\left(5^{\prime}-3^{\prime}\right)$ \\
\hline \multirow[t]{2}{*}{ GAPDH } & Forward & CCATCTTCCAGGAGCGAGAT \\
\hline & Reverse & TGCTGATGATCTTGAGGCTG \\
\hline \multirow[t]{2}{*}{ KRT6A } & Forward & TCACCGTCAACCAGAGTCTC \\
\hline & Reverse & GAACCTTGTTCTGCTGCTCC \\
\hline \multirow[t]{2}{*}{ E-cadherin } & Forward & ACGCATTGCCACATACACTC \\
\hline & Reverse & GGTGTTCACATCATCGTCCG \\
\hline \multirow[t]{2}{*}{ TIMP-2 } & Forward & TTCAAAGGGCCTGAGAAGGA \\
\hline & Reverse & TCAGGCTCTTCTTCTGGGTG \\
\hline \multirow[t]{2}{*}{ MMP-2 } & Forward & CAGCCCTGCAAGTTTCCATT \\
\hline & Reverse & GTTGCCCAGGAAAGTGAAGG \\
\hline \multirow[t]{2}{*}{ MMP-9 } & Forward & GAGACTCTACACCCAGGACG \\
\hline & Reverse & GAAAGTGAAGGGGAAGACGC \\
\hline \multirow[t]{2}{*}{$\beta$-Catenin } & Forward & CTTGTGCGTACTGTCCTTCG \\
\hline & Reverse & AGTGGGATGGTGGGTGTAAG \\
\hline \multirow[t]{2}{*}{ LEF-1 } & Forward & ACGAGCACTTTTCTCCAGGA \\
\hline & Reverse & ATAGCTGGATGAGGGATGCC \\
\hline \multirow[t]{2}{*}{ TCF-4 } & Forward & TCCTCCGATGTCCACTTTCC \\
\hline & Reverse & CCTGCTGAGAGAGATGGAGG \\
\hline
\end{tabular}

MMP, matrix metalloproteinase; E, epithelial; TIMP2, tissue inhibitor of metalloproteinase-2; LEF-1, lymphoid enhancer binding factor 1; KRT6A, keratin 6A; TCF-4, T-cell specific factor 4.

for $30 \mathrm{~min}$ and centrifuged at $1 \times 10^{4} \mathrm{x}$ g at $4^{\circ} \mathrm{C}$ for $20 \mathrm{~min}$. Next, the proteins in supernatant were quantified using BCA protein assay kit (Pierce; Thermo Fisher Scientific, Inc.). A total of $20 \mu \mathrm{g}$ protein was first loaded to each well that contained $12 \%$ SDS-PAGE and then separated and transferred onto polyvinylidene fluoride membranes (Thermo Fisher Scientific, Inc.). Next, the membranes were blocked in 5\% non-fat dry milk for $1 \mathrm{~h}$ at room temperature and incubated with specific primary antibodies overnight at $4^{\circ} \mathrm{C}$. Finally, the membranes were incubated with secondary antibodies conjugated with horseradish peroxidase: Anti-rabbit IgG, HRP-linked Antibody (Cell Signaling Technology, Inc., Danvers, MA, USA; cat. no. 7074, $1: 5,000)$ for $1 \mathrm{~h}$ at room temperature. GAPDH was treated as a loading control. The proteins were detected by enhanced chemiluminescence detection reagents (Pierce; Thermo Fisher Scientific, Inc.) and analyzed by Bio-Rad ChemiDoc XRS densitometry with Image Lab ${ }^{\mathrm{TM}}$ Software version 4.1 (Bio-Rad Laboratories, Inc., Hercules, CA, USA). The primary antibodies used were as follows: Rabbit anti-KRT6A (Abcam, Cambridge, UK; cat. no. ab18586; 1:200), E-cadherin (Abcam; cat. no. ab15148; 1:500), TIMP-2 (Abcam; cat. no. ab180630; 1:1,000), MMP-2 (Abcam; cat. no. ab92536; 1:1,000), MMP-9 (Abcam; cat. no. ab38898; 1:1,000), $\beta$-catenin (Abcam; cat. no. ab16051; 1:5,000), LEF-1 (Abcam; cat. no. ab22884; 1:500), TCF-4 (Abcam; cat. no. ab185736; 1:1,000) and GAPDH (Abcam; cat. no. ab9485; 1:2,000).

Statistical analysis. SPSS software (version 20.0; IBM Corps, Armonk, NY, USA) was used for data analysis. Three replications were conducted in each assay. Data were expressed with the mean \pm standard deviation. The data were analyzed by one-way analysis of variance and Dunnett t's test. $\mathrm{P}<0.05$ was considered to indicate a statistically significant difference.

\section{Results}

KRT6A is upregulated in NPC cells. The expression levels of KRT6A in the nasopharyngeal epithelial cell line (NP69, as control) and NPC cell lines (C666-1, 5-8F and SUNE-1) were measured. The results of RT-qPCR and western blotting demonstrated that the mRNA and protein levels of KRT6A were significantly upregulated in all detected NPC cells, among which the value was the highest in the SUNE-1 cells $(\mathrm{P}<0.01$; Fig. 1). Therefore, SUNE-1 cells were selected for the following gene modification experiments.

KRT6A silencing inhibits the cell viability of NPC cells. KRT6A-siRNA was transfected into NPC SUNE-1 cells using the liposome method. RT-qPCR and western blot assays analysis demonstrated that the mRNA and protein levels of KRT6A were significantly decreased in the siKRT6A group, compared with those of the Cotl and mock groups $(\mathrm{P}<0.01$; Fig. 2A-C). CCK-8 assay results demonstrated that cell viabilities in the siKRT6A group was inhibited compared with the two groups, Cotl and mock $(\mathrm{P}<0.01$; Fig. 2D).

KRT6A silencing inhibits cell metastasis and invasion of NPC cells. Cell metastasis and invasive abilities of SUNE-1 NPC cells prior to and following performing KRT6A silencing were measured by scratch wound healing and Transwell assays, respectively following $24 \mathrm{~h}$ of cell growth. The wound distance siKRT6A group were significantly increased compared with the Cotl and mock groups, indicating that KRT6A silencing inhibited cell metastasis of SUNE-1 NPC cells $(\mathrm{P}<0.01$; Fig. 3A and B). In addition, the invasion rates of SUNE-1 NPC cells significantly decreased in the siKRT6A group, suggesting that KRT6A silencing inhibited cell invasion of SUNE-1 NPC cells $(\mathrm{P}<0.01$; Fig. 3C and D).

KRT6A silencing attenuates EMT in NPC cells. EMT is the major mechanism of cell metastasis and invasion, Therefore, the epithelial marker E-cadherin and matrix degrading enzyme-associated factors were measured. RT-qPCR demonstrated though the mRNA and protein expression levels of E-cadherin and TIMP-2 were significantly promoted in the siKRT6A group $(\mathrm{P}<0.01)$, the mRNA and protein expression levels of MMP-2 and MMP-9 were significantly reduced compared with those in both the Cotl and mock groups ( $\mathrm{P}<0.01 ;$ Fig. 4).

KRT6A silencing inhibits the Wnt/ $\beta$-catenin pathway in NPC cells. As the Wnt/ $\beta$-catenin pathway strongly affects tumorigenesis, the effect of KRT6A silencing on the Wnt/ $\beta$-catenin pathway was investigated using RT-qPCR and western blot assays. The results demonstrated that both the mRNA and protein levels of $\beta$-catenin, LEF-1 and TCF-4 were significantly reduced in the siKRT6A group, compared with those in both the Cotl and mock groups $(\mathrm{P}<0.01$; Fig. 5).

Activation of the Wnt/ $\beta$-catenin pathway reverses the effect of $s i-K R T 6 A . \mathrm{LiCl}$ was used to activate the Wnt/ $\beta$-catenin 
A

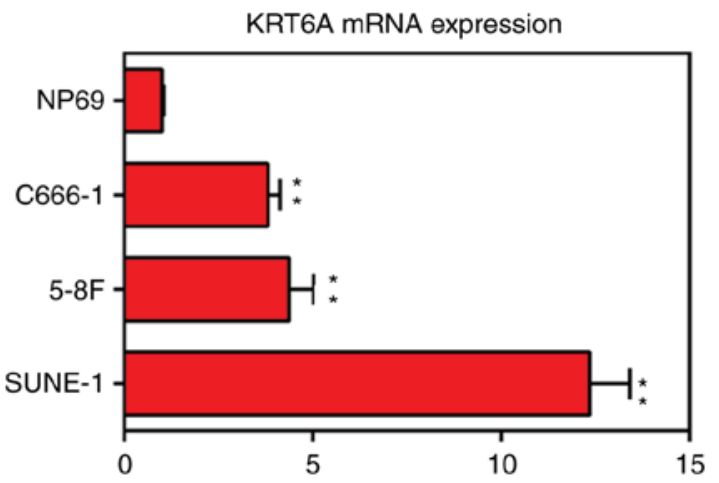

B

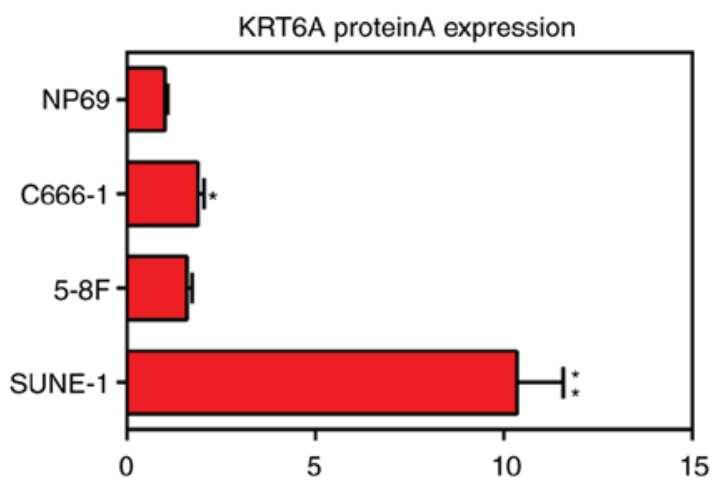

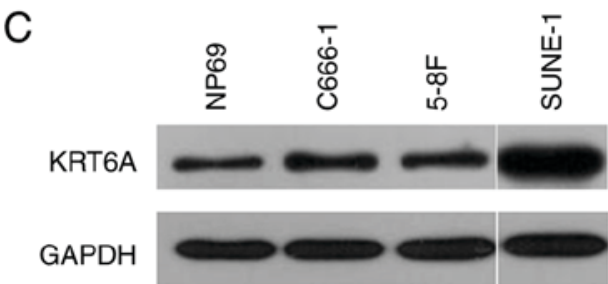

Figure 1. KRT6A is upregulated in NPC cells. (A) The mRNA expression levels of KRT6A were significantly upregulated in NPC cells (C666-1, 5-8F and SUNE-1), compared with normal nasopharyngeal epithelial cell line (NP69). (B) The protein levels of KRT6A were significantly upregulated in NPC cells and (C) and the western blot is presented. ${ }^{* *} \mathrm{P}<0.01$ vs. the NP69 group. KRT6A, keratin 6A; NPC, nasopharyngeal carcinoma.

A

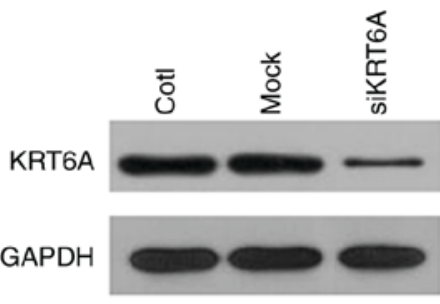

B

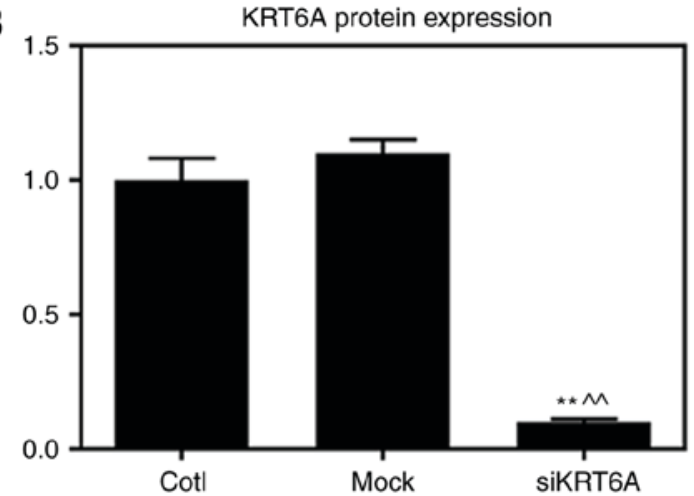

C

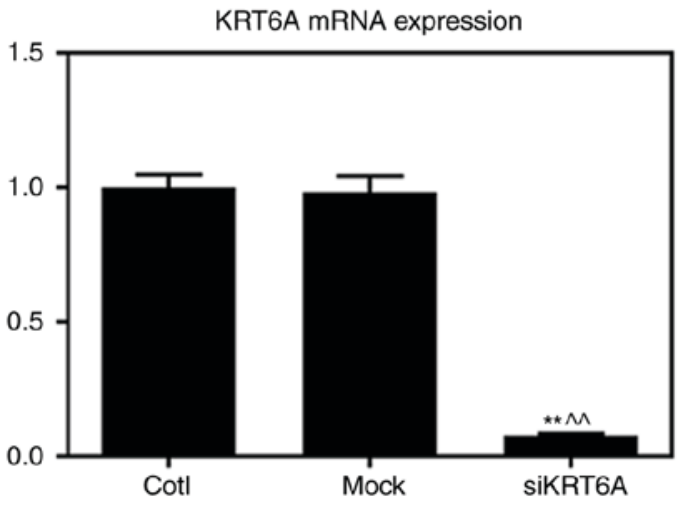

D

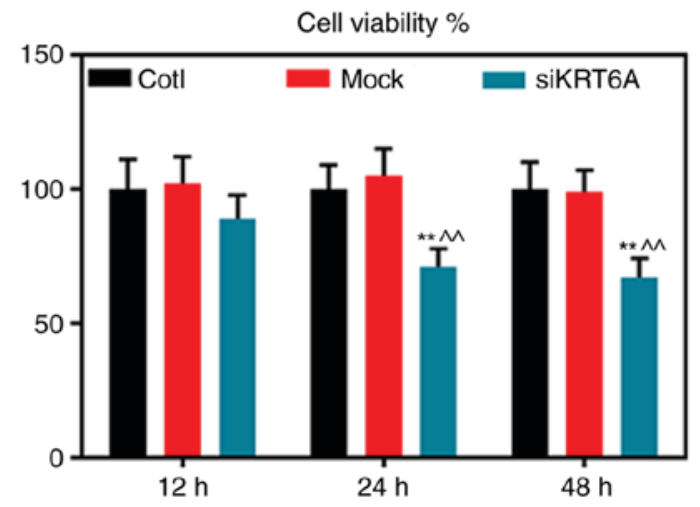

Figure 2. KRT6A silencing inhibits the cell viability of NPC cells. (A) The western blot is demonstrated and (B) the protein levels of KRT6A were demonstrated to decrease in the siKRT6A group. (C) The mRNA levels of KRT6A decreased in the siKRT6A group. (D) Cell viabilities in the siKRT6A group were inhibited at 24 and 48 h. ${ }^{* *} \mathrm{P}<0.01$ vs. the Cotl group and ${ }^{\wedge} \mathrm{P}<0.01$ vs. the mock group. KRT6A, keratin $6 \mathrm{~A}$; NPC, nasopharyngeal carcinoma; si, small interfering; Cotl, nontreatment group.

pathway and determined the association between the Wnt//-catenin pathway and si-KRT6A. It was observed that decreased expression of MMP2/9 caused by si-KRT6A was then rescued by LiCl. By contrast, the expression of TIMP2 was significantly decreased in LiCl+si-KRT6A group compared with the si-KRT6A group ( $\mathrm{P}<0.05$; Fig. 6). This suggested that the effect of si-KRT6A on NPC cells may be largely dependent on the inactivation of the Wnt/ $\beta$-catenin pathway. 
A

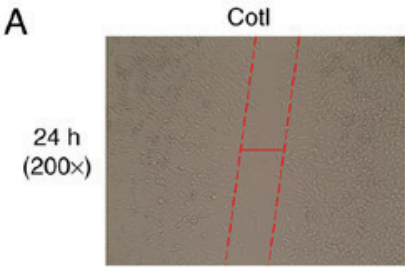

C

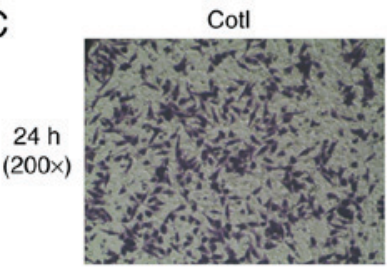

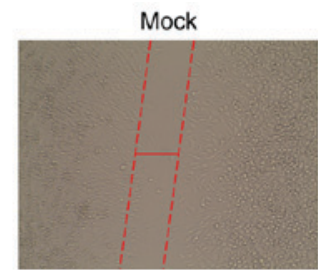

Mock

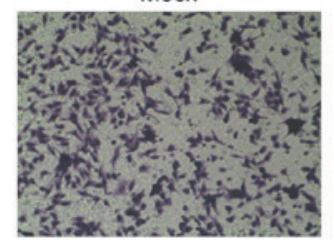

SiKRT6A

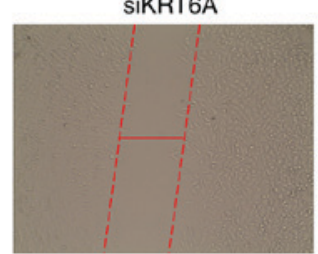

SIKRT6A

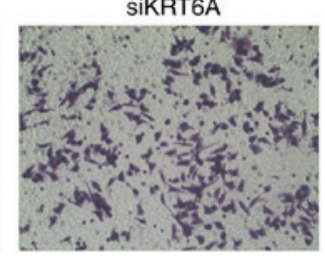

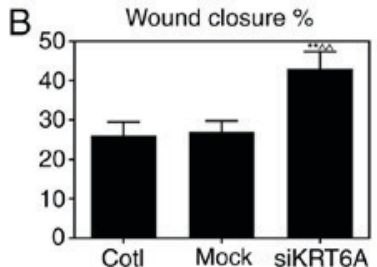

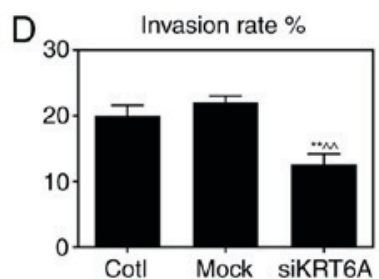

Figure 3. KRT6A silencing inhibits cell metastasis and invasion of NPC cells. KRT6A silencing inhibited cell metastasis of SUNE-1 NPC cells as detected by (A) a scratch wound healing assay and (B) the statistical analysis is presented. KRT6A silencing inhibited cell invasion of SUNE-1 NPC cells, detected by (C) a Transwell assay and (D) the statistical analysis is presented. ${ }^{* *} \mathrm{P}<0.01$ vs. the Cotl group and ${ }^{\wedge} \mathrm{P}<0.01$ vs. the mock group. KRT6A, keratin $6 \mathrm{~A}$; NPC, nasopharyngeal carcinoma; Cotl, nontreatment group.
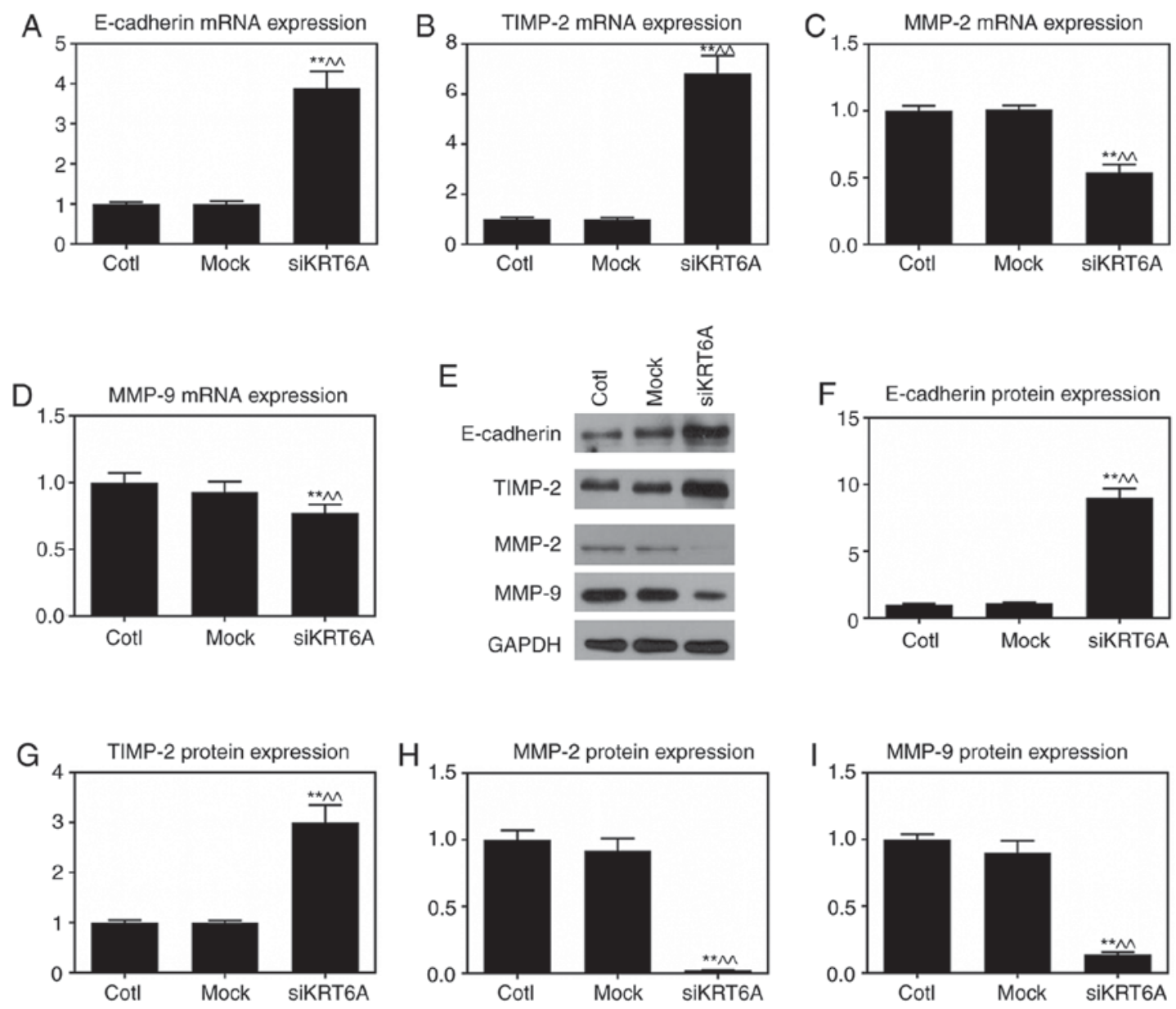

Figure 4. KRT6A silencing attenuates epithelial mesenchymal transition in NPC cells. (D) The mRNA expression levels of (A) E-cadherin and (B) TIMP-2 were significantly promoted in the siKRT6A group, while those of (C) MMP-2 and (D) MMP-9 were markedly reduced. (E) The protein levels of (F) E-cadherin and (G) TIMP-2 were significantly promoted in siKRT6A group, while those of (H) MMP-2 and (I) MMP-9 were markedly reduced. * P $<0.01$ vs. the Cotl group and ${ }^{\wedge} \mathrm{P}<0.01$ vs. the mock group. MMP, matrix metalloproteinase; E, epithelial; TIMP2, tissue inhibitor of metalloproteinase-2; NPC, nasopharyngeal carcinoma; si, small interfering; KRT6A, keratin 6A; Cotl, nontreatment group.

\section{Discussion}

NPC is widely regarded as an undifferentiated squamous cell carcinoma that is characterized by distant metastasis $(17,33)$. KRT6A is a biomarker unique to squamous cells and the correct expression of keratin genes is the basis for maintaining 

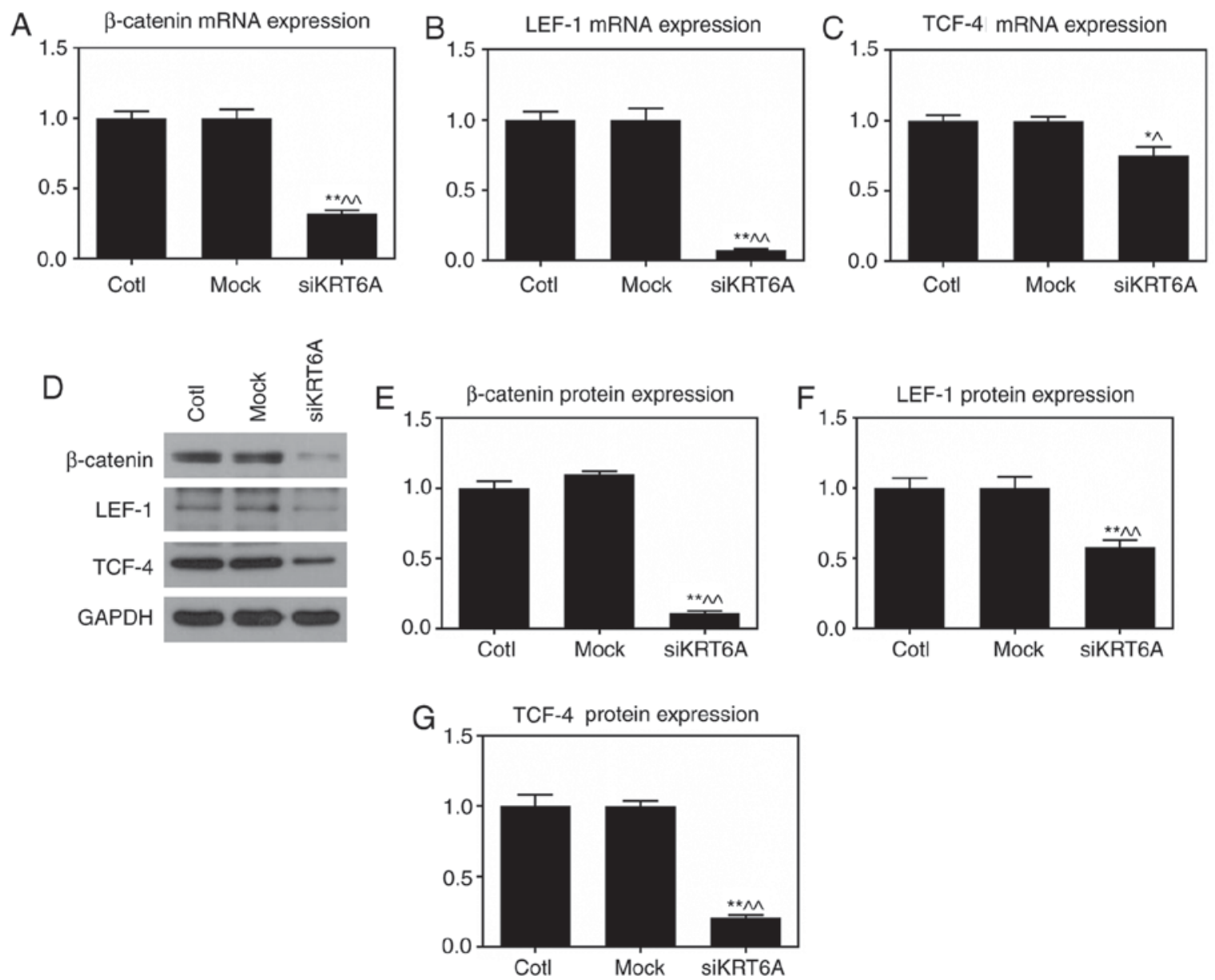

Figure 5. KRT6A silencing inhibits the Wnt/ $\beta$-catenin pathway in NPC cells. The mRNA expression levels of (A) $\beta$-catenin, (B) LEF-1 and (C) TCF-4 were significantly reduced in the siKRT6A group. (D) The protein levels of (E) $\beta$-catenin, (F) LEF-1 and (G) TCF-4 were significantly reduced in the siKRT6A group. ${ }^{* *} \mathrm{P}<0.01$ vs. the Cotl group and ${ }^{\wedge} \mathrm{P}<0.01$ vs. the mock group. TCF-4, T-cell specific factor 4; LEF-1, lymphoid enhancer binding factor 1; NPC, nasopharyngeal carcinoma; si, small interfering; KRT6A, keratin 6A; Cotl, nontreatment group.
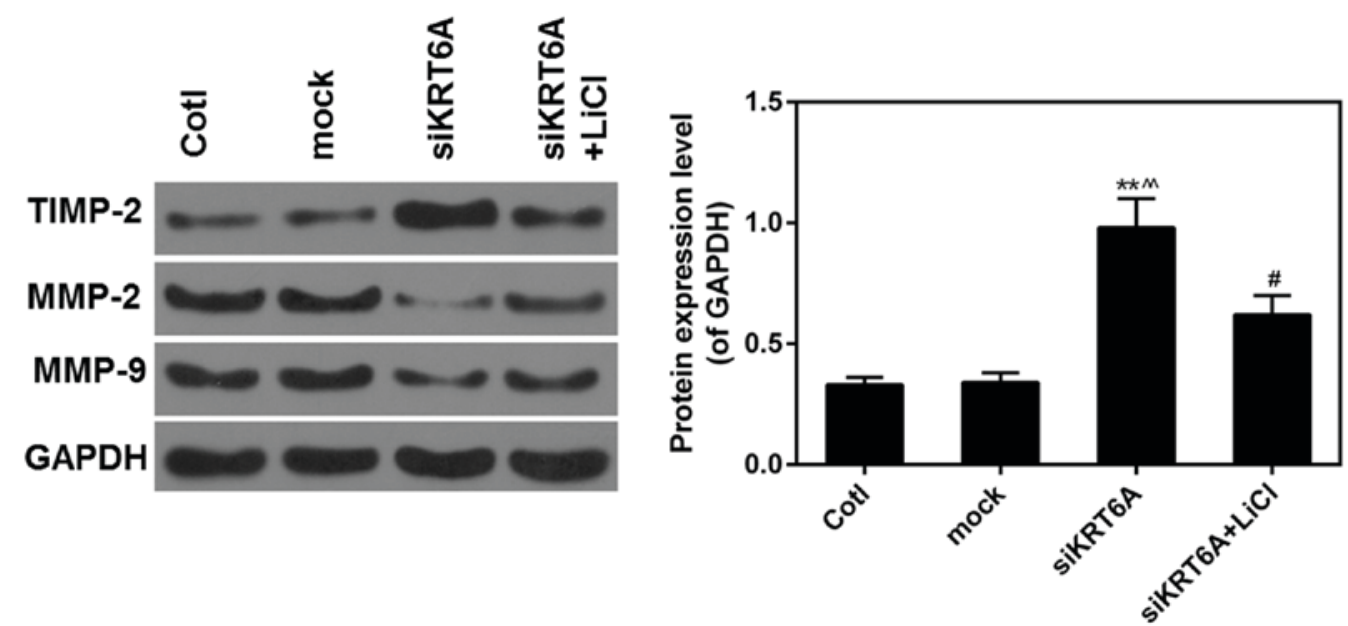

Figure 6. Wnt/ $\beta$-catenin pathway on the effect of KRT6A silencing. The expression of TIMP-2, MMP-2 and MMP-9 was detected using western blotting. ${ }^{* *} \mathrm{P}<0.01$ vs. the Cotl group, ${ }^{\wedge} \mathrm{P}<0.01$ vs. the mock group and ${ }^{\#} \mathrm{P}<0.05$ vs. the siKRT6A group. MMP, matrix metalloproteinase; TIMP2, tissue inhibitor of metalloproteinase-2; Cotl, nontreatment group; si, small interfering; KRT6A, keratin 6A.

the stability, and normal differentiation of epidermal cells (28). Therefore, it is interesting to know whether KRT6A affects cell growth and differentiation as well as distant metastasis in NPC. Therefore, this study focused on investigating the mechanisms of ectopic KRT6A in NPC. The expression levels of KRT6A in multiple NPC cell lines, which include C666-1, $5-8 \mathrm{~F}$ and SUNE-1 cells were first measured. It was demonstrated that the expression of KRT6A was high in SUNE-1. 
This may be explained by the fact that SUNE-1 is a type of poorly differentiated carcinoma cell. The poorly differentiated carcinoma cells have a high malignancy rate and can invade into distant organs and tissues. Therefore, KRT6A silencing was conducted in SUNE-1 cells and the effects of KRT6A silencing on cell viability, metastasis, and invasion of SUNE-1 NPC cells were determined.

As the process of EMT is critical to tumor metastasis and it has been reported to be involved in the modification process of numerous biomarkers (34), the expression levels of certain critical factors were detected in the EMT process. Being responsible for cell-cell adhesion in epithelial cells, E-cadherin is one of the most critical hallmarks of EMT $(35,36)$. Decrease of E-cadherin induces weakened epithelial characteristics and transition to the mesenchymal phenotype (37). In the present study, upregulated E-cadherin was tested when KRT6A was silenced in NPC cells. MMPs are the main proteolytic enzymes in the process of tumor metastasis, during which MMPs destroy the histological barrier for cell invasion (38-40). Type IV collagenases, including MMP-2 and MMP-9, are important in affecting tumor metastasis $(10,41,42)$. The present study demonstrated that KRT6A silencing suppressed the expression of MMP-2 and MMP-9, as well as facilitated the expression of TIMP-2 in NPC cells.

The Wnt/ $\beta$-catenin pathway is correlated with the EMT process in cancer. During the EMT process the stabilization and nuclear translocation of $\beta$-catenin is the critical events in Wnt/ $\beta$-catenin pathway (43-46). LEF-1 and TCF-4, two key members of TCF family, mediate the regulation of $\beta$-catenin-dependent transcription factors (47-50). To investigate the effect of KRT6A on the canonical $\beta$-catenin/TCF pathway in NPC, alterations of the expression of $\beta$-catenin, LEF-1 and TCF-4 were identified when KRT6A was silenced in NPC cells. The results proved that KRT6A silencing inhibited the expression of $\beta$-catenin, LEF-1 and TCF-4 so as to inactivate $\beta$-catenin/TCF pathway. In addition, the activation of $\mathrm{Wnt} / \beta$-catenin pathway, which was caused by $\mathrm{LiCl}$, reversed the effect of KRT6A silencing by regulating the expression of TIMP2 and MMP2/9. These results suggested that KRT6A silencing may produce its anti-tumor effect largely by inhibiting the $\mathrm{Wnt} / \beta$-catenin pathway.

The present study demonstrated the anti-tumor role of KRT6A silencing in NPC cells. However, it is equally significant to further investigate the effect of KRT6A through upregulating its expression, as well as to confirm the role of KRT6A in animal experiments.

In conclusion, KRT6A silencing suppressed cell viability, invasion and metastasis of NPC cells via $\beta$-catenin/TCF pathway. Therefore, KRT6A may be a novel biomarker in the diagnosis and treatment of NPC. Further research in vivo is required in order to provide more evidence that illustrates the role of KRT6A.

\section{Acknowledgements}

Not applicable.

\section{Funding}

No funding was received.

\section{Availability of data and materials}

All data generated or analyzed during this study are included in this published article.

\section{Authors' contributions}

CC performed all the experiments. HS contributed to the conception of the study and wrote the manuscript. All authors read and approved the final manuscript.

\section{Ethics approval and consent to participate}

Not applicable.

\section{Patient consent for publication}

Not applicable.

\section{Competing interests}

The authors declare that they have no competing interests.

\section{References}

1. Liu TF: Recent developments in diagnosis and treatment of nasopharyngeal carcinoma. Keio J Med 40: 59-62, 1991.

2. Wyatt DE, Brooker DS, Connolly JH and Coyle PV: Prognostic value of Epstein-Barr virus serology in patients with nasopharyngeal carcinoma. J Infect 26: 171-175, 1993.

3. Wang Y, Shen C, Lu X and Hu C: The incidence and prognosis of nasopharyngeal carcinoma patients with family history. Oncotarget 8: 97323-97330, 2017.

4. Wei KR, Zheng RS, Zhang SW, Liang ZH, Li ZM and Chen WQ: Nasopharyngeal carcinoma incidence and mortality in China, 2013. Chin J Cancer 36: 90, 2017.

5. Lu TX: Advance in diagnosis and management of local recurrent nasopharyngeal carcinoma. Ai Zheng 23: 230-234, 2004 (In Chinese).

6. Xia L, Zhang W, Zhu L and Yu G: Abnormally expressed long non-coding RNAs in nasopharyngeal carcinoma: A meta-analysis. Clin Lab 64: 585-595, 2018.

7. Chan AT, Leung TW, Kwan WH, Mok TS, Yeo W, Lai M and Johnson PJ: Phase II study of Temodal in the treatment of patients with advanced nasopharyngeal carcinoma. Cancer Chemother Pharmacol 42: 247-249, 1998.

8. Li Y, Ou X, Shen C, Xu T, Li W and Hu C: Patterns of local failures and suggestions for reduction of clinical target volume for nasopharyngeal carcinoma patients without cervical lymph node metastasis. Onco Targets Ther 11: 2545-2555, 2018.

9. Qi XK, Han HQ, Zhang HJ, Xu M, Li L, Chen L, Xiang T, Feng QS, Kang T, Qian CN, et al: OVOL2 links stemness and metastasis via fine-tuning epithelial-mesenchymal transition in nasopharyngeal carcinoma. Theranostics 8: 2202-2216, 2018.

10. Ogasawara N, Kudo T, Sato M, Kawasaki Y, Yonezawa S, Takahashi S, Miyagi Y, Natori Y and Sugiyama A: Reduction of membrane protein CRIM1 decreases E-cadherin and increases claudin-1 and MMPs, enhancing the migration and invasion of renal carcinoma cells. Biol Pharm Bull 41: 604-611, 2018.

11. Tuo Z, Zhang J and Xue W: LncRNA TP73-AS1 predicts the prognosis of bladder cancer patients and functions as a suppressor for bladder cancer by EMT pathway. Biochem Biophys Res Commun 499: 875-881, 2018.

12. Shi B, Wang Y and Yin F: MALAT1/miR-124/Capn4 axis regulates proliferation, invasion and EMT in nasopharyngeal carcinoma cells. Cancer Biol Ther 18: 792-800, 2017.

13. Li S, Zhang X, Zhang R, Liang Z, Liao W, Du Z, Gao C, Liu F, Fan $\mathrm{Y}$ and Hong $\mathrm{H}$ : Hippo pathway contributes to cisplatin resistant-induced EMT in nasopharyngeal carcinoma cells. Cell Cycle 16: 1601-1610, 2017. 
14. Zuo LL, Zhang J, Liu LZ, Zhou Q, Du SJ, Xin SY, Ning ZP, Yang J, Yu HB, Yue WX, et al: Cadherin 6 is activated by Epstein-Barr virus LMP1 to mediate EMT and metastasis as an interplay node of multiple pathways in nasopharyngeal carcinoma. Oncogenesis 6: 402, 2017.

15. Wu SL, Li YJ, Liao K, Shi L, Zhang N, Liu S, Hu YY, Li SL and Wang Y: 2-Methoxyestradiol inhibits the proliferation and migration and reduces the radioresistance of nasopharyngeal carcinoma CNE-2 stem cells via NF- $\kappa \mathrm{B} / \mathrm{HIF}-1$ signaling pathway inactivation and EMT reversal. Oncol Rep 37: 793-802, 2017

16. Hu Y, Qi MF, Xu QL, Kong XY, Cai R, Chen QQ, Tang HY and Jiang W: Candidate tumor suppressor ZNF154 suppresses invasion and metastasis in NPC by inhibiting the EMT via Wnt/3-catenin signalling. Oncotarget 8: 85749-85758, 2017.

17. Wang G, Zhang L, Zhou Y, Sun Q, Xu H, Cai F, Xiang P, Chen Z and Jiang H: KAI1/CD82 genetically engineered endothelial progenitor cells inhibit metastasis of human nasopharyngeal carcinoma in a mouse model. Med Sci Monit 24: 3146-3152, 2018

18. Aouf S, Laribi A, Gabbouj S, Hassen E, Bouaouinaa N, Zakhama A and Harizi H: Contribution of Nitric oxide synthase 3 genetic variants to nasopharyngeal carcinoma risk and progression in a Tunisian population. Eur Arch Otorhinolaryngol: 13 Feb, 2019 (Epub ahead of print).

19. Chua HH, Kameyama T, Mayeda A and Yeh TH: Cancer-specifically re-spliced TSG101 mRNA promotes invasion and metastasis of nasopharyngeal carcinoma. Int $\mathrm{J}$ Mol Sci 20: pii: E773, 2019.

20. Petersson F: EBV-associated non-keratinizing nasopharyngeal carcinoma with prominent spindled cell and whorling patterns: A previously unreported histological variant in a patient presenting with dermatomyositis. Head Neck Pathol: 13 Feb, 2019 (Epub ahead of print)

21. Xiao H, Guo Y, Yi J, Xia H, Xu H, Yuan L, Hu P, Yang Z, He Z, $\mathrm{Lu} \mathrm{H}$ and Deng $\mathrm{H}$ : Identification of a novel keratin 9 missense mutation in a chinese family with epidermolytic palmoplantar keratoderma. Cell Physiol Biochem 46: 1919-1929, 2018.

22. Jacob JT, Coulombe PA, Kwan R and Omary MB: Types I and II keratin intermediate filaments. Cold Spring Harb Perspect Biol 10: pii: a018275, 2018.

23. Komori T, Ono M, Hara ES, Ueda J, Nguyen HTT, Nguyen HT, Yonezawa T, Maeba T, Kimura-Ono A, Takarada T, et al: Type IV collagen $\alpha 6$ chain is a regulator of keratin 10 in keratinization of oral mucosal epithelium. Sci Rep 8: 2612, 2018.

24. Deo PN and Deshmukh R: Pathophysiology of keratinization. J Oral Maxillofac Pathol 22: 86-91, 2018.

25. Gu LH and Coulombe PA: Keratin function in skin epithelia: A broadening palette with surprising shades. Curr Opin Cell Biol 19: 13-23, 2007.

26. Chang HH, Dreyfuss JM and Ramoni MF: A transcriptional network signature characterizes lung cancer subtypes. Cancer 117: 353-360, 2011.

27. Forrest CE, Casey G, Mordaunt DA, Thompson EM and Gordon L: Pachyonychia congenita: A spectrum of KRT6a mutations in australian patients. Pediatr Dermatol 33: 337-342, 2016.

28. Cammarata-Scalisi F, Natsuga K, Toyonaga E, Nishie W, Shimizu H, Avendaño A, Araque D, Da Silva G, Bellacchio E and Callea M: Early severe pachyonychia congenita subtype PC-K6a with a novel mutation in the KRT6A gene. J Eur Acad Dermatol Venereol 31: e94-e96, 2017.

29. Chai AW, Cheung AK, Dai W, Ko JM, Ip JC, Chan KW, Kwong DL, Ng WT, Lee AW, Ngan RK, et al: Metastasis-suppressing NID2, an epigenetically-silenced gene, in the pathogenesis of nasopharyngeal carcinoma and esophageal squamous cell carcinoma. Oncotarget 7: 78859-78871, 2016.

30. Zhang P, Zhang L, Liu H, Zhao L, Li Y, Shen JX, Liu Q, Liu MZ and $\mathrm{Xi}$ M: Clinicopathologic characteristics and prognosis of tongue squamous cell carcinoma in patients with and without a history of radiation for nasopharyngeal carcinoma: A matched case-control study. Cancer Res Treat 49: 695-705, 2017.

31. Clement-Lacroix P, Ai M, Morvan F, Roman-Roman S, Vayssière B, Belleville C, Estrera $\mathrm{K}$, Warman ML, Baron R and Rawadi G: Lrp5-independent activation of Wnt signaling by lithium chloride increases bone formation and bone mass in mice. Proc Natl Acad Sci USA 102: 17406-17411, 2005.

32. Livak KJ and Schmittgen TD: Analysis of relative gene expression data using real-time quantitative PCR and the 2(-Delta Delta C(T)) method. Methods 25: 402-408, 2001.

33. Wang P, Shen N, Liu D, Ning X, Wu D and Huang X: TRIM24 siRNA induced cell apoptosis and reduced cell viability in human nasopharyngeal carcinoma cells. Mol Med Rep 18 369-376, 2018
34. Setyaningsih WAW, Arfian N, Suryadi E, Romi MM, Tranggono U and Sari DCR: Hyperuricemia induces Wnt5a/Ror2 gene expression, epithelial-mesenchymal transition, and kidney tubular injury in mice. Iran J Med Sci 43: 164-173, 2018.

35. Yang F, Wei K, Qin Z, Liu W, Shao C, Wang C, Ma L, Xie M, Shu Y and Shen H: MiR-598 suppresses invasion and migration by negative regulation of derlin-1 and epithelial-mesenchymal transition in non-small cell lung cancer. Cell Physiol Biochem 47: 245-256, 2018

36. Zheng P, Li H, Xu P, Wang X, Shi Z, Han Q and Li Z: High lncRNA HULC expression is associated with poor prognosisand promotestumor progression by regulating epithelialmesenchymal transition in prostate cancer. Arch Med Sci 14 679-686, 2018

37. Zhang Y,Zhao L, Wang L, Yang X, Zhou A and Wang J: Placenta growth factor promotes epithelial-mesenchymal transition-like changes in ARPE-19 cells under hypoxia. Mol Vis 24: 340-352, 2018.

38. Chiu YJ, Hour MJ, Jin YA, Lu CC, Tsai FJ, Chen TL, Ma H, Juan YN and Yang JS: Disruption of IGF-1R signaling by a novel quinazoline derivative, HMJ-30, inhibits invasiveness and reverses epithelial-mesenchymal transition in osteosarcoma U-2 OS cells. Int J Oncol: 16 Mar, 2018 (Epub ahead of print).

39. Ciaramella V, Della Corte CM, Di Mauro C, Tomassi S, Di Maro S, Troiani T, Martinelli E, Bianco R, Cosconati S, Pierantoni R, et al: Antitumor efficacy of Kisspeptin in human malignant mesothelioma cells. Oncotarget 9: 19273-19282, 2018.

40. Fu X, Zhu Y, Zheng B, Zou Y, Wang C, Wu P, Wang J, Chen H, Du P, Liang B and Fang L: KIFC1, a novel potential prognostic factor and therapeutic target in hepatocellular carcinoma. Int J Oncol 52: 1912-1922, 2018

41. Jiang Y, Jiao Y, Liu Y, Zhang M, Wang Z, Li Y, Li T, Zhao X and Wang D: Sinomenine hydrochloride inhibits the metastasis of human glioblastoma cells by suppressing the expression of matrix metalloproteinase-2/-9 and reversing the endogenous and exogenous epithelial-mesenchymal transition. Int J Mol Sci 19: pii: E844, 2018.

42. Kur-Piotrowska A, Bukowska J, Kopcewicz MM, Dietrich M, Nynca J, Slowinska M and Gawronska-Kozak B: Foxn1 expression in keratinocytes is stimulated by hypoxia: Further evidence of its role in skin wound healing. Sci Rep 8: 5425, 2018.

43. Bush BM, Brock AT, Deng JA, Nelson RA and Sumter TF: The Wnt/ $\beta$-catenin/T-cell factor 4 pathway up-regulates high-mobility group A1 expression in colon cancer. Cell Biochem Funct 31: 228-236, 2013

44. Narasipura SD, Henderson LJ, Fu SW, Chen L, Kashanchi F and Al-Harthi L: Role of $\beta$-catenin and TCF/LEF family members in transcriptional activity of HIV in astrocytes. J Virol 86: 1911-1921, 2012

45. Yu XW, Xu Q, Xu Y, Gong YH and Yuan Y: Expression of the E-cadherin $/ \beta$-catenin/tcf- 4 pathway in gastric diseases with relation to Helicobacter pylori infection: Clinical and pathological implications. Asian Pac J Cancer Prev 15: 215-220, 2014.

46. Zhang J, Huang K, Shi Z, Zou J, Wang Y, Jia Z, Zhang A, Han L, Yue X, Liu N, et al: High $\beta$-catenin/Tcf-4 activity confers glioma progression via direct regulation of AKT2 gene expression. Neuro Oncol 13: 600-609, 2011.

47. Qian L, Zhang W, Zhang P, Lei B, Wang X, Wang M, Bai J and He A: The anti-apoptosis effect of MLAA-34 in leukemia and the $\beta$-catenin/T cell factor 4 protein pathway. Am J Transl Res 7: 2270-2278, 2015

48. Sun J, Jia Z, Li B, Zhang A, Wang G, Pu P, Chen Z, Wang Z and Yang W: MiR-19 regulates the proliferation and invasion of glioma by RUNX3 via $\beta$-catenin/Tef- 4 signaling. Oncotarget 8: 110785-110796, 2017.

49. Wang K, Wang X, Zou J, Zhang A, Wan Y, Pu P, Song Z, Qian C, Chen Y, Yang S and Wang Y: miR-92b controls glioma proliferation and invasion through regulating Wnt/beta-catenin signaling via Nemo-like kinase. Neuro Oncol 15: 578-588, 2013.

50. Xie J, Xiang DB, Wang H, Zhao C, Chen J, Xiong F, Li TY and Wang XL: Inhibition of Tcf-4 induces apoptosis and enhances chemosensitivity of colon cancer cells. PLoS One 7: e45617, 2012.

This work is licensed under a Creative Commons Attribution-NonCommercial-NoDerivatives 4.0 International (CC BY-NC-ND 4.0) License. 